\title{
Meeting Report:
}

\section{USP Workshop on In Vitro Testing for Meeting Future Challenges for Veterinary Dosage Forms}

Margareth R. C. Marques

U.S. Pharmacopeia, Rockville, MD, USA

T he United States Pharmacopeial Convention hosted the USP Workshop on In Vitro Testing for Meeting Future Challenges for Veterinary Dosage Forms at its headquarters in Rockville, MD, USA, on March 14 and 15, 2016.

The workshop planning committee was composed of James Demuth, Ph.D., Chair, USP General ChaptersDosage Forms Expert Committee; Jennifer Johansson, J.D., Generic Animal Drug Alliance (GADA); Craig Parks, M.S., D.V.M., Animal Health Institute (AHI); Julie Lorenz, Ph.D., Zoetis; David Long, M.S., Ph.D., Member, USP General Chapters-Dosage Forms Expert Committee; Marilyn Martinez, Government Liaison, USP Expert Panel Solubility Criteria for Veterinary Drugs, FDA Center for Veterinary Medicine; Eden Bermingham, M.S., D.V.M., FDA Center for Veterinary Medicine; Mai Huynh-Government Liaison, USP General Chapters, Dosage Forms Expert Committee, FDA Center for Veterinary Medicine; Morgan Puderbaugh, USP Scientific Liaison, Chemical Medicines; and Margareth Marques, USP Principal Scientific Liaison, General Chapters.

The oral route of administration is one of the most used for veterinary products. When developing formulations that will be applied to different animal species, it is important to consider the physiological differences among these species. The main objective of this workshop was to discuss the biopharmaceutics aspects of veterinary dosage forms. The first day was dedicated to the discussion of the challenges when working with different animal species and the physiological differences for dogs, cattle, pigs, horses, and poultry that may impact the oral administration of drug products. On the second day, several cases studies of the development of dissolution tests for unusual drug products, such as medicated feeds and chewable and palatable dosage forms, were presented.

The workshop opened with a presentation by John Mauger (member of the USP General Chapters-Dosage Forms Expert Committee), who gave an overview of the USP revision process. He discussed how the USP expert committees and expert panels work on the revision of monographs and general chapters. Any revisions are posted in the USP Pharmacopeial Forum Web site (http://www.usppf.com) for a period of 90 days for public comments. The comments received are evaluated by the appropriate groups and, if approved, are incorporated in the text that will become official. More information is available at http://www.usp.org/usp-nf/developmentprocess/submit-new-revised-usp-nf-monographs.

An overview of the USP project for solubility evaluation of veterinary drug products was presented by Marilyn Martinez (Government Liaison, USP Expert Panel Solubility Criteria for Veterinary Drugs, FDA Center for Veterinary Medicine). The Biopharmaceutics Classification System (BCS) developed by Dr. Gordon Amidon in 1990-1991 (1) resulted in the FDA Guidance for Industry Waiver of In Vivo Bioavailability and Bioequivalence Studies for Immediate-Release Solid Oral Dosage Forms Based on a Biopharmaceutics Classification System (new draft posted on May/2015 at http://www.fda.gov/ucm/groups/fdagovpublic/@fdagov-drugs-gen/documents/document/ ucm070246.pdf). The BCS is grounded in estimates of human jejunal permeability and drug solubility. Oral drug absorption is controlled by three dimensionless numbers: Dose Number $\left(D_{0}\right)$ : maximum proportion of dose that will be dissolved; Dissolution Number $\left(D_{n}\right)$ : GI residence time versus time needed to achieve complete dissolution; and Absorption Number $\left(A_{n}\right)$ : relationship between $\mathrm{Gl}$ residence time and time for membrane permeation. The in vivo solubility considerations are that the smaller the $D_{0}$, the more likely that the API will be fully solubilized in the GI tract; and the greater the $D_{\mathrm{n}}$, the more likely that the API will be fully solubilized in vivo. Dr. Amidon was invited to help explore the extension of BCS to animal health. The discussions were summarized in two publications $(2,3)$. These publications were used as the starting point for the work of the USP Expert Panel Solubility Criteria for Veterinary Drugs, initially focusing on dogs $(4,5)$. Since the effective permeability $\left(P_{\text {eff }}\right)$ reflects several variables (transcellular and paracellular movement and active transport), it was concluded that 
it is difficult to extrapolate $P_{\text {eff }}$ across species. Therefore, the focus of the discussion was directed to considering the criteria appropriate for describing drug solubility in veterinary species. A definition of "fully soluble" in veterinary species was developed starting with dogs and cattle, the most common veterinary patients in smalland food-animal practices (6). Based on the discussions during the 2012 USP workshop (7), it was decided to focus the studies on species-appropriate conditions for testing thermodynamic solubility and not to discuss dose number $\left(D_{0}\right)$. The ability to accurately measure the aqueous solubility of a material is affected by the following: (1) physicochemical properties of the material (e.g., surface area, particle size, crystal form); (2) properties of the solubility media (e.g., pH, polarity, surface tension, added surfactants, cosolvents, salts); and (3) control of the solubility measurement parameters (e.g., temperature, time, agitation method) (8). These properties and parameters, together with the composition of possible media that try to simulate gastrointestinal conditions for humans, dogs, and cattle, will be described and discussed in the new USP General Chapter <1236> Solubility Measurements that will be published in Pharmacopeial Forum 43(2) [March-April 2017] (http://www.usppf.com) for public comments.

Dr. Martinez made a comparison between solubility and dissolution evaluations and discussed possible relationships. A distinction must be made to the in vivo effects due to the API solubility (dose number) and time to dissolve (dissolution number) and to the formulation dissolution characteristics (rate and extent). The API solubility is a function of its physicochemical characteristics and how it was processed. The product dissolution is a function of the API characteristics, the excipients present, and the manufacturing process. Solubility is the amount of a substance that will dissolve in a fixed amount of solvent at a given temperature and pressure. Saturation solubility $\left(C_{s}\right)$ is the maximum amount of solute that can be dissolved within a given volume of solute. Cs can be expressed as the maximum mass in solution per unit volume. It is not necessary to know the species $\mathrm{Gl}$ fluid volume to define $C_{\mathrm{s}}$, but it is necessary to define $D_{0}$. The smaller $D_{0}$, the more likely it is that the API will be fully solubilized in the GI tract. The greater the $D_{n}$, the more likely it is that the API will be fully solubilized in vivo. The factors that affect $C_{s}$ include intrinsic solubility, $\mathrm{p} K_{\mathrm{a}}$, crystal characteristics, stereochemistry, salt form, $\mathrm{pH}$, buffer composition, common-ion effect, ionic strength, cosolvents, surfactants, and temperature. In vitro dissolution test conditions are intended to reflect formulation and processing effects on drug release. The rate-limiting factor determining release of the API in vivo should likewise be the rate-limiting factor in vitro. When this is not the case, the dissolution results can lead to false conclusions. Formulation effects cannot be adequately characterized if the in vitro release is constrained by $C_{s}$. Another important factor to be considered is $\mathrm{Gl}$ fluid dynamics. A recent study (9) showed that water in the stomach and small intestine is distributed in small pockets. This has important implications with respect to in vivo dissolution of oral dosage forms.

The challenges when working with different animal species were discussed by Steven Sutton (University of New England). The sources of variability that could impact results from bioequivalence studies can be divided into two areas: (1) pharmaceutics and pharmacokinetics and (2) physiology. Under the former are the broad areas of disintegration, dissolution, active pharmaceutical ingredient (API) stability, permeability, and bioavailability, while under the latter are gastric $\mathrm{pH}$ and emptying, small intestine transit, large intestine transit, passive permeability, active permeability, transporters, and intestinal metabolism. Beyond these are the myriad of differences that are associated with the numerous breeds of cats and dogs.

\section{Sources of Variability Among Dogs}

Some of the important variables attributed to the stomach are the size of the pylorus aperture and the size of the dog, stomach contractions among different breeds, as well as hydrodynamic flow and fluid volume. Food is an oftenoverlooked source of variability. Fat alone stimulates the secretion of bile acids; calories and fats both slow gastric emptying and can buffer stomach $\mathrm{pH}$.

\section{pH}

The average $\mathrm{pH}$ value measured in the stomach is often reported, but it can be misleading. For example, if in $50 \%$ of the observations the $\mathrm{pH}$ is 1 and in the other $50 \%$, the $\mathrm{pH}$ is 7 , the average of $\mathrm{pH} 4$ does not describe it very well. What help would data have on a $\mathrm{pH}$-sensitive formulation? The $\mathrm{pH}$ value may also differ by location in the stomach: anterior $\mathrm{pH}>$ posterior $\mathrm{pH}$. Studies of stomach $\mathrm{pH}$ with a telemetric capsule demonstrate that as the location of the telemetric capsule changes during the experiment, the $\mathrm{pH}$ changes. These studies therefore observe the $\mathrm{pH}$ over a reasonable length of time.

\section{Gastric Emptying}

In the fasted canine, a dosage form can be too large to empty before the migrating myoelectric complex ("housekeeper waved") sweeps by-"size matters." Tablet strength also matters, especially since a tablet that 
is too soft will be crushed in the comparatively strong contractive forces of the stomach. In the fed canine, the composition ( $\mathrm{CHO}$, proteins, etc.) of the meal determines the extent of delay in gastric emptying.

\section{GI Transit Time (GITT)}

The literature can be summarized that in the fasted beagle dog, gastric emptying is approximately $1 \mathrm{hr}$, small intestinal transit time (SITT) ranges from 0.3 to $2 \mathrm{~h}$, and the colon transit time ranges from 24 to $48 \mathrm{~h}$. The fed dog similarly has variability

\section{Intestinal Transporters in Canines}

Uptake transporters such as intestinal PEPT1 and OCT1 decreased from small intestine to colon (similar to human). Efflux transporters such as intestinal MDR1 increased along the small intestine and decreased in colon (human increased from duodenum to colon). Intestinal MRP1and BCRP increased from small intestine to colon (similar to human). Aside from a study on collies, very little research has examined the difference in transporter expression among breeds.

\section{Small Intestinal Metabolism in Canines}

Intestinal CYP2D15 levels in the dog may be >CYP3A12, CYP3A26 and increase from small intestine to colon, whereas in humans, CYP2D6 decreases from the small intestine to the colon. Intestinal CYP3A26 and CYP3A12 were constant along the gut in the dog, whereas human CYP3A4 decreased from the small intestine to the colon. Intestinal expression of UGT1A6 in the dog was similar to that in humans.

\section{Sources of Variability Among Cats Esophageal Blockage}

The esophageal transit time (ETT) is less than $0.5 \mathrm{~min}$ in a cat with a normal ETT. However, if a capsule gets stuck in the cat, the ETT can be greater than 5 min. Once a cat has an episode, it is likely to be repeated in future studies. The pharmacokinetics or therapeutic effects are usually observed with immediate-release formulations of BCS Class I compounds. Other reports state that the normal range of ETT in cats is $0.5-5 \mathrm{~min}$. When No.4 capsules were orally administered to cats ("dry swallow"), the capsules lodged in the esophagus of over half of the cats for $>4$ min. Esophageal transits shortened to under 0.5 $\min$ in $90 \%$ of the cases following administration with just $6 \mathrm{~mL}$ of water.

\section{Gastric Emptying: Variability, Fasted Cats}

The primary factors of interest in the fed condition that impact gastric emptying of the API in cats are hairballs, ETT, and tablet fragments. Hairballs consist of hair/fur consumed during grooming and not digested or passed into the feces. The consequences of hairballs are usually only a mild discomfort for the cat, resulting in some gagging and coughing to regurgitate the obstruction. The impact of the hairball on gastrointestinal transit before it grows to a size that induces vomiting is not known. If emptied from the stomach, the trichobezoar can cause intestinal obstruction. Hairballs are not usually a problem for short-haired cats. Some cats have a differently shaped stomach (anatomy) or contractions (physiology) that may result in more frequent hairballs. If the fur accumulates and partially obstructs the passage of food through the pylorus, the cat may vomit. A gastric obstruction could significantly delay gastric emptying.

\section{When Would Delayed ET or GE Be an Issue?}

Delayed ET or GE may be an issue for BCS Class I drugs, high first-pass extracted drugs, drugs that exhibit nonlinear bioavailability or are acid labile with a low fraction absorbed (BCS Class II), or even those with a low aqueous solubility (BCS Class IV).

\section{Case Study}

In vitro dissolution test conditions were similar for the test and reference items (USP Apparatus 1; $0.1 \mathrm{~N} \mathrm{HCl,} \mathrm{pH}$ $1.2,900 \mathrm{~mL} ; 100 \mathrm{RPM}$ ), and 18 cats were administered either the test or reference item in a two-way crossover study. The study failed to demonstrate bioequivalence with maximum concentration in the plasma $\left(C_{\max }\right)$. However, two cats had anomalies. In the first cat, the reference product was considerably lower than those of the mean test or reference $C_{\max }$. The test $C_{\max }$ was similar to those of the mean reference and mean reference $C_{\text {max }}$ In the second subject, the test concentration-time profile was considerably lower than either the mean test or reference data. Repeat administration to the cats with apparent anomalies may or may not result in a repeat of the anomalies. The conclusion is that the variability is due to the physiology, not the pharmaceutics. By playing the numbers (i.e., larger sample size), will you eventually prove $\mathrm{BE}$ ?

The assessment and interpretation of solubility for canine oral drug products were discussed by Mark Papich (North Carolina State University, USP Expert Panel Solubility Criteria for Veterinary Drugs). To select the appropriate conditions to evaluate drug solubility in dogs, it is important to consider anatomic, functional, and physiologic differences between different species (dogs versus humans) (10) and among different breeds (intraspecies). Compared with humans, dogs have a shorter intestinal transit time that is affected by feeding, additional surface area for absorption, an increased bile 
salt secretion that can play an important role in increasing solubility of poorly water-soluble drugs, and higher intestinal $\mathrm{pH}$ resulting in better absorption of weak bases. Some of the factors that influence the gastric residence of dosage forms in dogs are time of dosing relative to the phase of the interdigestive migrating myoelectric current, the sieving properties of the pylorus, the presence or absence of food, the inherent crushing force of the stomach, API properties, and dosage form characteristics such as tablet hardness (11). Oral absorption of drug between dogs and humans do not correlate. There is sparse or no data in the literature for the oral absorption, permeability $\left(P_{\text {eff }}\right)$, and $\mathrm{p} K_{\mathrm{a}}$ values in dogs. There is no drug standard for dogs such as metoprolol for humans (there is a recommendation for the use of bisoprolol instead), and there is no standardized volume for the calculation of $D_{\mathrm{n}}(250 \mathrm{~mL}$ versus $35 \mathrm{~mL})$. Most of the in vivo studies in the literature were done using beagle dogs, but there is no information to show that these results can be applied to other canine breeds. The test conditions proposed to evaluate the solubility of APIs in dogs are test the maximum dose $(\mathrm{mg} / \mathrm{kg}$ ) approved by FDA based on approved dose bands and use $10 \mathrm{~mL}$ of solvent, at $37^{\circ} \mathrm{C}$, within the $\mathrm{pH}$ values of $1.2,4.5$, and 7.5 using buffer solutions normally used to evaluate API solubility for human drugs.

Vivek Fellner (North Carolina State University) discussed the rumen and drug interactions. The rumen is a complex ecosystem with a high diversity of microorganisms (about $10^{12} / \mathrm{mL}$ ). It has complicated metabolic reactions, diurnal variations, and dietary perturbations. The rumen volume can be in excess of $200 \mathrm{~L}$; it has a slow and incomplete mixing; it has an intermittent inflow and outflow; and it has periodic regurgitation and eructation. The rumen contains enzymes with hydrolytic, reduction, decarboxylation, dealkylation, and dehalogenation reaction mechanisms that may inactivate or activate various compounds including drugs. The fermentation in the rumen produces short chain fatty acids (SCFA), mainly acetate and propionate, which are an energy source. The rumen and its flora can have an effect on the drug being administered, and the drug can have an effect on the rumen microorganisms (12).

The feline and canine gastrointestinal physiologies were discussed by Andrea Fascetti (University of California). The Gl tracts of dogs and cats are similar with the exception of length. The GI tract small and large intestines are shorter in cats than in dogs. The length influences the amount of time food is in the Gl tract and the duration of the digestion process. The Gl tract in both species has a large surface area (jejunum surface area is about 50 $\mathrm{cm}^{2}$ in cats and $54 \mathrm{~cm}^{2}$ in dogs). The feline gastric mucosa is uniform, whereas the canine has two distinct areas: the proximal stomach has thinner mucosa with distinct glands, and the distal stomach has thicker mucosa and less distinct glands. The saliva $\mathrm{pH}$ is $7.3-7.8$ in dogs and around 7.5 in cats. Dogs and cats lack salivary $\alpha$-amylase. The $\mathrm{pH}$ of the feline stomach is $2.5 \pm 0.07$. Gastric pepsin is very important for cats because it initiates meat digestion and is very active in collagen digestion. The gastric halfemptying times in cats are 22-25 min (fasted) to 449 min (canned diet), and for dogs the time is between 72 and $240 \mathrm{~min}$. The feline intestinal $\mathrm{pH}$ increases due to bicarbonate in pancreatic fluid and bile: stomach $2.5 \pm$ 0.07 , duodenum $5.7 \pm 0.5$, jejunum $6.4 \pm 0.5$, and ileum $6.0 \pm 0.8$. The carbohydrate digestion occurs in the small intestine. Starch is digested by $\alpha$-amylase and $\alpha$-dextrin. The four major brush border enzymes are glucoamylase (maltase), sucrase, $\alpha$-dextrinase (iso-maltase), and lactase ( $\beta$-galactosidase). The pancreatic concentration of amylase is three times higher than that in dogs. The absorption of lactose is limited by brush border hydrolysis. The concentration of microorganisms in the small intestine is about $1 \times 10^{4} \mathrm{CFU} / \mathrm{mL}$ in dogs and about $2.2 \times 10^{5}$ to $1.6 \times 10^{8} \mathrm{CFU} / \mathrm{mL}$ in cats (mostly anaerobes). The microbes influence the nutrient requirements and the diet can influence microbes. Cat taurine is an essential dietary requirement for feline health. Cats, unlike most mammals, are unable to manufacture taurine from other amino acids. Taurine is therefore crucial for a cat's health. Some factors that may impact nutrient digestibility in cats are food processing effects (particle size, modifications to processing equipment), feeding management practices (previous diet fed, amount of food fed, timing), animal factors (breed, age, gender, activity, physiological state), and housing and environmental factors (housing type, temperature, photoperiod, and caretaker-animal relationship).

Jerome del Castillo (Universite de Montreal) discussed the therapeutic use of drugs in swine with a focus on feed medication. The most preferable way of administering drugs to pigs is in the feed or water because of some constraints such as low worker/animals ratios and animal size, force, and wrestling. The major benefits of this method of administration are low stress for the animals and staff and the unlimited numbers of pigs that can be treated as long as they have free access to feed or water. Palatability may be an issue because the taste acuity is higher in pigs than in humans. Sick pigs do not eat or drink as often as healthy animals, and the oral bioavailability of the drug should be considered. Feed is 
a convenient drug dosing vehicle because the animal will spontaneously consume it; it attenuates the unpleasant taste of a drug, and most of the ingredients in the feed are generally recognized as safe. Most ingredients are vegetal (e.g., corn, soymeal), some are of animal origin (e.g., milk powder), and some industrial by-products from bakery, distillery, dairy, fishery activities are used as main ingredients or as additives. Feed may look like a simple drug-dosing vehicle, but there are some parameters that must be controlled. Particle size is from $1 \mathrm{~mm}$ to less than $100 \mu \mathrm{m}$ (in most cases from 700 to $800 \mu \mathrm{m}$ ); dry matter content is from 85-90\% in dry feed and from $20-30 \%$ in liquid feed. It is often pelleted, requiring finer grinding and heat/vapor processing, and water holding capacity to sponge the gastric fluids (e.g., soymeal has a water holding capacity of $5 \mathrm{~L} / \mathrm{kg}$ ). The mixing of gastric contents is very poor in pigs. The solid matter deposits in the greater gastric curve, the digestive secretions interact with only the lower periphery, and any ingested water runs over it. The drug is added to the feed as a premix. Several drug carriers can be used, and they can interact with the drug and determine its release kinetics. These premixes are often granulated to provide a more stable and homogeneous dispersion in the feed. The particle size, porosity, and hardness are parameters that must be controlled as they affect the "wettability" of the drug and, consequently, its release kinetics. The drug release rate from the feed-grade premixes can be affected by the feed, and the increasing $\mathrm{pH}$ in the $\mathrm{Gl}$ tract can precipitate them. Absorption kinetics profiles in pigs after administration of drugs in feed suggest a zero-order process. The parameters to consider when administering drugs to pigs in the drinking waters are variability of water supply among farms (influence on the $\mathrm{pH}$, hardness, etc.) and cleanliness of water tubing and dispensers at the farm (they are not cleaned on regular basis; there is accumulation of lime and organic material over time, and sometimes feed-grade premix is dispensed through water). To better understand how drugs may be released and absorbed in the swine Gl tract, an update on the knowledge of the digestive physiology in pigs is needed because most of the studies are old. Also, a more reasonable compromise between premix stability and drug release efficiency is needed. The formulations for premix need improvement based on swine physiology.

The GI physiology of horses was discussed by Jane Owens (Elanco Animal Health). Horses are herbivores that eat grass between 15 to $20 \mathrm{~h}$ per day. They have a small stomach and a large cecum, with hindgut fermentation. There is a continuous movement of ingest, and they produce about 20-25 kg of feces per day. The cardiac sphincter is a very strong one-way valve; there is no regurgitation. In contrast, the pyloric sphincter is mostly open, and duodenal regurgitation is common. Horses have a simple stomach, the smallest one in relation to body size of all domestic animals, with a volume between 8 and $15 \mathrm{~L}$ depending on the animal size. It secretes gastric acid continuously. The gastric residence time varies depending on feedstuff and amount, with an average between 2 and $6 \mathrm{~h}$. Fluids have a short retention time, but solid can be retained for more than $48 \mathrm{~h}$. The equine stomach is rarely empty; it empties when $2 / 3$ full. A 500-kg animal can produce $30 \mathrm{~L}$ of gastric juice in $24 \mathrm{~h}$. Bacterial fermentation is present. The gastric $\mathrm{pH}$ varies considerably with prandial state, time of the day, feedstuff, and region of the stomach. The $\mathrm{pH}$ is around 3.1 in the fed state, 1.55 in the unfed state, between 6 and 7 at the cardiac sphincter, and between 1 and 2 at the pyloric sphincter. The equine small intestine has a length of about $22 \mathrm{~m}$; it has a rapid transit time, typically $30 \mathrm{~cm} /$ $\mathrm{min}$, and the $\mathrm{pH}$ at the initial portion of the small intestine is around 6.7. The cecal and colonic fermentation accounts for large portion of energy needs. The cecum is about $1 \mathrm{~m}$ in length, has a capacity of up to $36 \mathrm{~L}$ and a pH of about 7.0. The large intestine/colon is about $8 \mathrm{~m}$ long, and it has a $\mathrm{pH}$ of about 7.4 . Between 75 and $85 \%$ of the $\mathrm{GI}$ transit is spent in the large intestine with an average transit time of about $35 \mathrm{~h}$. A horse consumes about $15 \mathrm{~kg} /$ day of food, half of it as forage. Drug binding or drug adsorption can occur with feedstuffs; some examples are phenylbutazone, ampicillin, and flunixin. Significant prandial effects may occur on the pharmacokinetics of certain drugs such as fluxinin and moxidectin. Some of the challenges with orally dosing horses are their size and weight; they don't always like being dosed; they are unpredictable and react quickly; their heads can be weapons; their teeth are dangerous; all four of their feet can be weapons; they have prehensile lips and a very mobile tongue; and they have a large oral cavity that can retain feedstuff. The most common methods of dosing horses are by oral administration (paste, gels, tablets, sublingual gels) and in the feed (powders, granules, tablets). The major concerns when administering any oral dosage forms to horses are if the dosage form was ingested/swallowed; if the animal caretaker was hurt; if the animal is eating; how the next dose will be administered. Tablets for use in horses must be very small and soluble in aqueous fluids. Pastes, powders, and granules must be developed in a way that guarantees their homogeneity and stability. Other critical parameters are segregation, static charge, flow, and dust. The major concerns with sublingual gels are imprecise dosing and possible oral absorption. 
Jeff Buhr (U.S. National Poultry Research Center) gave a detailed description of the chicken alimentary tract and the factors that influence feed passage. The major parts of the alimentary tract are crop, gizzard, duodenum, small intestine, cecum, large intestine, and cloaca. Chickens are monogastric omnivores. They swallow feed whole; there is no chewing as they do not have teeth. They have a prehensile beak with no lips or cheeks. The palatine choanal cleft cannot suck liquids. The chicken alimentary tract and transit time are shorter than those of mammals. There are peristaltic and antiperistaltic contractions among segments (crop $\leftrightarrow$ duodenum; gizzard $\leftrightarrow$ mouth; cloaca $\leftrightarrow$ cecum). There is no absorption of nutrients or drugs at the mouth, esophagus, or gizzard. The hydrolysis of starch and proteins starts at the crop. Its $\mathrm{pH}$ varies depending on diet and animal age/stage: it is higher than 6 in laying hens and between 4.5 and 5.9 in chicks on starter feed. The crop $\mathrm{pH}$ is higher if the animal is taking pelleted rather than mash feeds, decreases from 6 to 5.3 after one week on feed boilers, and increases with extended time of feed. The crop fills when the gizzard muscles are contracting. The amount and frequency of feed intake depends on the light exposure periods. The gizzard is a mechanical stomach. It has a luminal cuticle layer to protect from acid exposure and grinding abrasion. It can hold $5-10 \mathrm{~g}$ of material and has a pH between 2 and 3.5. The duodenum is the first segment of small intestine, it is a site for digestion (95\% of fat digestion) and absorption (vitamin B6), and it has a $\mathrm{pH}$ above 6 . The jejunum is the longest segment, about $124 \mathrm{~cm}$. It has a $\mathrm{pH}$ of about 6.4 and contains digestive enzymes (maltase, isomaltase, sucrose, enterokinase, lipase, and peptidase). The large intestine has about the same diameter as the small intestine but it is shorter in length. The cecum has some fiber digestion and vitamin synthesis. It has a $\mathrm{pH}$ of about 6.6 and a retention time between 2 and $12 \mathrm{~h}$. The colon has a pH of about 6.4 and a retention time of about $50 \mathrm{~min}$. The cloaca is the common exit for the digestive, urinary, and reproductive tracts. The major sites of absorption in the chicken are small intestines and ceca for amino acids; distal jejunum and ileum for lipids; small intestines and ceca for volatile fatty acids; and duodenum, jejunum, and ileum for carbohydrates. Supplements and vitamins are administered by incorporation in the feed. They must withstand manufacturing, processing (freeflowing), and distribution (temperature and moisture stability) and have good compatibility with the other components of the formulation. They must have a good thermostability during pelleting. These supplements and added substances may be microencapsulated to have a targeted delivery. Some products can be administered to chickens through the drinking water (e.g., vaccines and probiotics).

The second-day presentations started with an overview on dissolution testing by Mansoor Khan (Texas A\&M Health Science Center). Dissolution is an approved and validated method for measuring the rate of drug release from a dosage form. Its main applications are optimization of the product to ensure consistency in product development and stability assessment; routine assessment of product quality to ensure uniformity among production lots; assessment, in some cases, of "bioequivalence" from discrete batches of product; and prediction of in vivo availability after determination of an in vivo-in vitro correlation (IVIVC). He reported about 98 product recalls in the United States due to noncompliance with dissolution requirements. He emphasized that for a vast majority of dosage forms, dissolution is the only performance test without any substitute. He gave a brief description of each one of the dissolution apparatus described in the $\operatorname{USP}(13,14)$. A brief discussion of the definitions and application of $f_{1}$ and $f_{2}$ in the comparison of dissolution profiles was presented (15). He discussed what type of information and data can be obtained through the FDA Dissolution Methods Web site (16) and the USP Dissolution Methods database (17) and what data can be gathered using these two tools. For example, most of the dissolution tests are for tablets (67\%) and capsules $(23 \%)$, and most of the tests are for immediate release ( $77 \%$ for tablets and $74 \%$ for capsules). The most frequently used equipment for dissolution testing of tablets is the paddle and for capsules, the basket. He finalized his presentation with a discussion on dissolution results and in vivo data and how they could be used to better understand differences between the innovator and generic products using the examples of metoprolol succinate extended-release tablets and bupropion tablets.

The use of USP Apparatus 4 (flow-through cell) for in vitro method development was presented by Sunil Potdar. This equipment can be used for almost any type of dosage forms but can be very useful for complex parenteral products. The flow-through cell can be operated in the open-loop mode, where infinite sink conditions can be maintained, or in closed loop, ideal for low-dose dosage forms. Several different types of cells can be selected depending on the dosage form type. The steps to be followed during the development of a dissolution procedure using Apparatus 4 are development of the quantitative procedure (usually HPLC); determination of the drug substance solubility in different media to select 
the most appropriate one for dissolution determination; selection of the cell type and adapter; decision to work in a closed or open loop; optimization of the medium; optimization of the medium volume; optimization of the flow rate; optimization of glass bead size and amount; and evaluation of the discriminatory power of the test using different pilot formulations. Once the conditions that provide the most discrimination are selected, the following parameters are used for the method validation: accuracy, linearity and range, repeatability, intermediate precision, and specificity (18). The stability of all the solutions used in the dissolution procedure must be determined. Once the dissolution method has been validated it can be used in the comparison of dissolution profiles between test and reference products using, as an example, the calculations of $f_{1}$ and $f_{2}(15)$.

A case study was presented by Danna Mattocks (Tergus Pharma) on the development of dissolution procedures for Type A medicated feed. Type A medicated articles are concentrated forms of animal drugs intended solely for further manufacture of other approved Type A medicated articles or Type B or C medicated feeds. Type A medicated articles cannot be fed directly to animals. They consist of one or more animal drugs with or without a carrier (e.g., calcium carbonate, rice hull, corn, gluten) and with or without other inactive ingredients. They can be prepared in dry or liquid form. They are sold to feed mills or livestock producers and are intended to be further diluted by mixing into feed before consumption by animals (19). Dissolution of medicated feed is a relatively new area of interest with consequently limited information available in the literature. There are neither well-established dissolution protocols nor regulatory guidance. Medicated feed is a bulk product containing insoluble excipients with limited aqueous dispersability. It may have variable doses, and the sample to be used in the dissolution test is typically measured in grams. This type of product presents unusual challenges. The determination of the dose depends on multiple factors such as animal species size, average daily intake, and duration of dosing. The typical quantity consumed is too large for dissolution testing using the compendial conditions. The definition of dissolution medium volume is another challenge because the gastric volume of large species is impossible to replicate and special equipment may be required to replicate the volumes in small species. Dense material that rapidly sinks to the bottom of the vessel may experience significant coning when USP Apparatus 2 is used. The dispersion characteristics of the product may significantly influence the selection of the dissolution conditions. The sample introduction technique may be critical.
Formulation wetting and clumping are also key factors to be considered as they can result in inadequate contact between sample and medium, limiting the amount of API released. The use of baskets (USP Apparatus 1) may help in uniformly dispersing the sample and increasing contact with dissolution media, but it will limit the sample size. Sample filtration is another challenge due to the high turbidity or high amount of insoluble excipients. Fine particles will require small pore-size filters. The presence of high concentrations of large particles can result in back pressure and clogged filters. It may be necessary to use prefilters. The use of larger pore cannula filters for sample collection should also be considered. The calculation of the amount of API dissolved can be complicated. It may be expressed as feed potency (grams of active/kg of product) or as a standard potency (units of activity/g). The selected calculation should ensure accurate representation of the reported sample strength and standard concentration. The release results may show greater variability than those observed for other oral solid dosage forms.

The next case study was presented by Timothy Priddy (Boehringer-Ingelheim) whose article on characterizing the in vitro drug exchange profile of a modified-release parenteral solution for veterinary use appears in this issue of Dissolution Technologies (20).

Elizabeth Cormier (Center for Veterinary Medicine/U.S. FDA) discussed the definitions and characteristics of tablets.

Chewable tablets: Formulated and manufactured to produce a pleasant-tasting residue in the mouth and to facilitate swallowing. Hard chewable tablets are typically prepared by compaction, typically utilizing mannitol, sorbitol, or sucrose as binders and fillers and containing colors and flavors to enhance their appearance and taste. Soft chewable tablets are typically made by a molding or extrusion process, frequently with more than $10 \%$ water to help maintain a pliable, soft product. Hard chewable tablets in veterinary medicine often have flavor enhancers like brewer's yeast or meat/fish-based flavors. Tablets for human use that include "chewable" in the title must be chewed or crushed prior to swallowing to ensure reliable release of the drug substance(s) or to facilitate swallowing. If tablets are designed such that they may be chewed (but chewing is not required for drug substance release or ease of swallowing), the title should not include a reference to "chewable." In that case, the product may still be described as chewable in the ancillary labeling statement. Tablets for veterinary use that are intended to be chewed will include Chewable in the title. However, it is understood that for veterinary products, it is not possible 
to ensure that tablets are chewed prior to ingestion. Chewable tablets may be broken into pieces and fed to animals that normally swallow treats whole.

Buccal tablets: Intended to be inserted in the buccal pouch, where the drug substance is absorbed directly through the oral mucosa. Few drug substances are readily absorbed in this way (e.g., nitroglycerin and certain steroid hormones).

Orally disintegrating tablets: Orally disintegrating tablets are intended to disintegrate rapidly within the mouth to provide a dispersion before the patient swallows the resulting slurry where the drug substance is intended for gastrointestinal delivery, absorption, or both. Some of these dosage forms have been formulated to facilitate rapid disintegration and are manufactured by conventional means or by lyophilization or molding processes. Further details may be found in the FDA guidance for orally disintegrating tablets (21).

Sublingual tablets: Sublingual tablets are intended to be inserted beneath the tongue, where the drug substance is absorbed directly through the oral mucosa. As with buccal tablets, few drug substances are extensively absorbed in this way, and much of the drug substance is swallowed and is available for gastrointestinal absorption.

Tablets for oral solution: Before administration, tablets for oral solution are intended to be solubilized in a liquid diluent. In some cases, they may also be chewed or swallowed.

Tablets for oral suspension: Tablets for oral suspension are intended to be dispersed in a liquid before administration as a suspension when either the drug substance or the excipients do not dissolve when dispersed in a liquid. In some cases, tablets for oral suspension may also be chewed or swallowed.

Tablet triturates: Small, usually cylindrical, molded, or compacted tablets. Tablet triturates traditionally were used as dispensing tablets to provide a convenient, measured quantity of a potent drug substance for compounding purposes, but they are rarely used today.

Effervescent tablets: Prepared by compaction and contain mixtures of acids (e.g., citric acid or tartaric acid) and carbonates or sodium bicarbonate in addition to the drug substance(s). Upon contact with water, these formulations release carbon dioxide, producing the characteristic effervescent action.

Hypodermic tablets: Molded tablets made from completely and readily water-soluble ingredients, formerly intended for use in making preparations for hypodermic injection. They may be administered orally or sublingually when rapid drug substance availability is required.

Tablets can also be categorized by the route of administration: oral, rectal, vaginal, and subcutaneous (implants).

Implants (pellets): An implant is a small tablet that is manufactured in the same way as a tablet. It is a non-oral dosage form that can remain in the body of the animal for up to 170 days. They can also be either immediate- or modified release.

Tablets can also be classified according to their release mechanism:

- Immediate-release tablets: No deliberate effort has been made to modify the drug substance release rate. Typically, it dissolves in the $\mathrm{Gl}$ contents with no delay or prolonging of the drug dissolution or absorption.

- Modified-release tablets: The release of the drug is altered by time or location. They can be classified as:

- Extended-release tablets are formulated in such a manner as to make the active ingredient available over an extended period of time following ingestion. A zero-order release of drug is needed for extended release, meaning that the rate of drug release is independent of drug concentration. Mechanisms may include matrix systems (hydrogel, polymeric matrix, wax matrix), polymeric film-coating, ion-exchange resins, and multiparticulate systems (granulates, pellets, beads).

- Delayed-release tablets are generally designed to delay the release of the API until the tablet has passed through the stomach. Delayed-release tablets are desired when the API may be destroyed or inactivated by the gastric juice or irritate the gastric mucosa. This can be accomplished using enteric coating or prodrugs.

Tablets can be manufactured by various processes such as direct compression, dry granulation, wet granulation, extrusion, and molding.

Typically the parameters for monitoring the quality of 

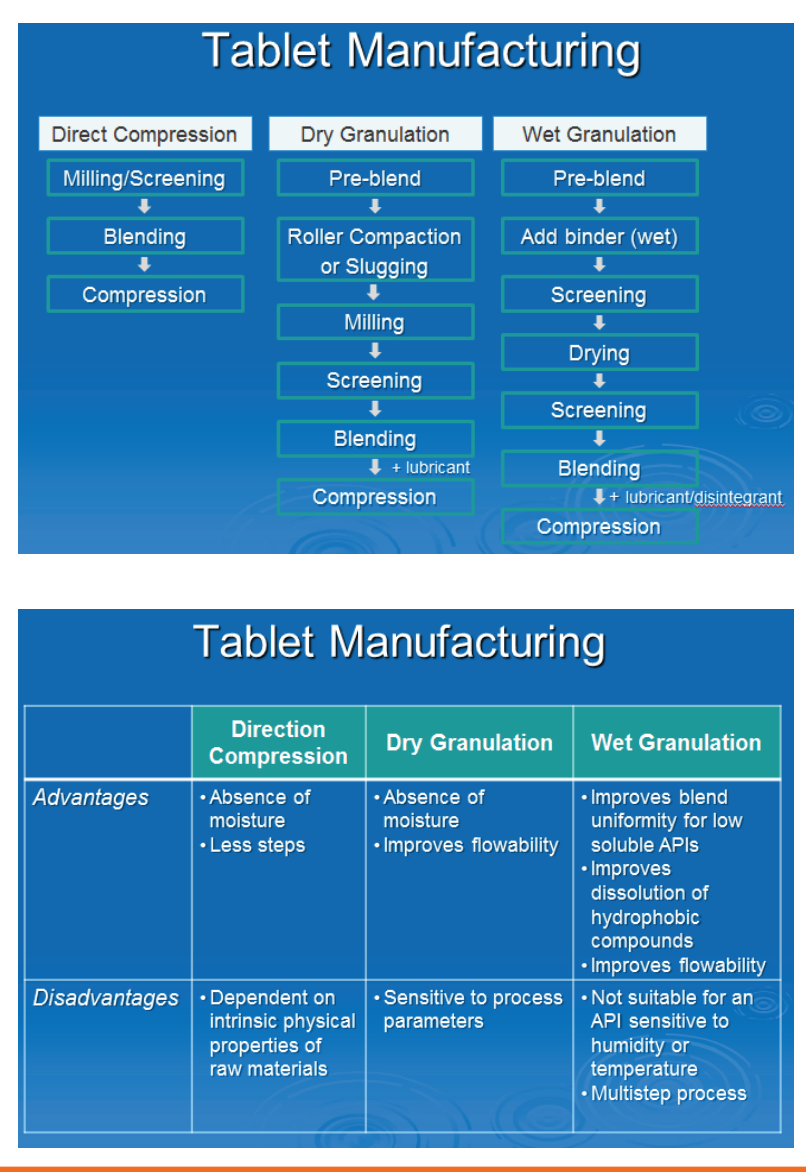

tablets are description, appearance, identification, assay, impurities, and microbial limits. The major critical quality attributes for tablets are dissolution/disintegration, hardness/tensile strength, friability (should not exceed $1 \%$ of tablet weight), content uniformity, and water content. For tablets that are scored (22), it is important to evaluate on split tablets the uniformity of dose, friability, and loss of mass at both ends of hardness, and dissolution. In the case of tablets containing low doses, it is important to demonstrate blend uniformity.

An overview on the development of chewable dosage forms was presented by Julie Lorenz (Zoetis). The industry favors chewable tablets mainly for their ease of administration to companion animals resulting in improved compliance with dosing regimens. There are other considerations that make the development of chewable tablets complex. There are many different dog sizes contributing to the need for a wide range of doses. The market is global, and not all regions have the same preferences for dosage forms. Chewables are intended to encourage full consumption and free-choice acceptance. The pet owner can hand the medicine to the animal or place it in a bowl and it will be fully eaten. There are many choices to be made such as a palatability label statement, desired texture, appearance, flavor, size, and shape. Once the product profile is defined, the API characteristics of solubility and permeability, taste, stability, and dose must be taken into account. It may be necessary to use taste-masking to overcome the bad taste of the API. A good taste can be imparted with the use of flavors and palatability enhancers. The formulation type is selected to meet the desired product profile. Chewable tablets can be hard or soft. To develop successful chewable tablets, consideration should be given to the combination of palatability, stability, and biopharmaceutics. Beyond the API characteristics, there are unique excipients that must be considered such as source of the flavor (animal, non-animal, artificial), palatability enhancers, sweeteners, and especially for dogs, salts as bitterness blockers. If the API has a bad taste, some options are chemical taste-masking or simply overpowering a bad taste with a good one. Physical tastemasking can involve coating or complexing the API so that it is blocked from interaction with the receptors on the tongue. Hard chewable tablets can be manufactured by wet granulation, dry granulation, or direct compression. Soft chewable tablets can be made by extrusion or by molding. There are some techniques that can be used to help with optimizing chewable tablet formulations. The electronic tongue is an array of nonspecific chemical sensors with cross sensitivity to different tastes that can be used to assess flavors or formulations. Data analysis is usually done using multivariate statistics. An early correlation with in vivo data is required to know which differences are meaningful. It can assess placebo versus active formulations to assess taste-masking effectiveness. The electronic tongue can also be used to get an early reading on API bitterness. Newer technologies include receptor-based systems with sensors that have been developed based on species and taste-specific receptors. This technology is emerging in the areas of both pet food and human food. An alternative technique to electronic tongues, when targeting physical taste-masking, is the use of early time points in a dissolution profile. This technique is widely reported in human pharmaceutical development. To evaluate the effectiveness of the tastemasking approach, the amount of drug release in the first 2 min of the profile can be determined and correlated to in vivo palatability data. Once the correlation is developed, formulations can be screened to ensure the taste-masking effectiveness. The in vivo palatability test can be designed as a single test article acceptance test or a multiple test article acceptance test. The selection of the dogs that will participate in the study must be as diverse as possible. A structure between feeding and offering treats must be 
defined, including offering treats at the same time each day, and must include exercise and play as well as periods of animal training offering treats at a set time and a diverse offering of treats and placebos. Once the palatability is fairly well achieved, it is necessary to verify that the formulation options do not impact bioavailability. A good approach is to check bioavailability relatively early in the project, especially if the formulation includes physical taste-masking. Regarding the formulation stability, consideration must be given to formation of impurities/ degradation products, water content because flavor and palatability enhancers often add moisture, and potential hardening or changes in texture over time.

Kevin White (Elanco Animal Health) discussed a case study on the development of a dissolution test for chewable formulations for companion animals. The product is a fixed combination with two drug substances in very different doses, one with API 1/API 2 at 1000 $\mathrm{mg} / 15 \mathrm{mg}$, and the other with API 1/API 2 at $100 \mathrm{mg} / 1.5$ $\mathrm{mg}$. Dissolution behavior was initially evaluated using buffers of pH 3.5, 4, 4.5, and 5 with different proportions of surfactant. The solubilities of the crystalline form and the spray-dried dispersion were determined using surfactant in the concentrations of $0.05 \%, 0.1 \%, 0.15 \%$, $0.2 \%, 0.25 \%, 0.3 \%$, and $0.35 \%$. The spray-dried dispersion is a single-phase, amorphous molecular dispersion of drug in a polymer matrix. The API and the polymer are dissolved in an organic solvent, and the solution is spraydried to produce an amorphous dispersion. One of the major advantages of this type of formulation is the enhancement in the oral bioavailability of poorly soluble compounds. Experimental design was used to develop the strategy for the selection of the most appropriate dissolution conditions.

Palatability testing was discussed by Ann Stohlmann (Center of Veterinary Medicine/U.S. FDA). Palatable is defined as acceptable to the taste; as sufficiently agreeable in flavor to be eaten. It is synonymous with acceptability. Palatability testing is not specifically required by regulation. The veterinary $\mathrm{ICH}$ guideline for effectiveness testing of anthelmintics in canine (23) recommends palatability testing for applicable oral anthelmintics. Palatability information should be presented in a factual manner consistent with the data. Section 502(a) of the Federal Food, Drug, and Cosmetic Act states that information on the package labeling must not be misleading. Terms such as "flavored" and "chewable" are based on the description of the drug products and its components, and they are not dependent on palatability testing. Palatability testing should be done in the target population and is generally conducted during the clinical field study. Studies done in a small number of animals or in laboratory animals may be useful for exploratory purposes. Palatability may be assessed as the percentage of doses that fall into the following categories applicable to the dosage form, in the order of most to least palatable:

- The dose is voluntarily consumed when offered by hand or in a bowl (for tablets).

- The dose is voluntarily consumed when offered in or on food (any dosage form).

- The dose is voluntarily swallowed when placed in the oral cavity (for liquids, pastes, and gels).

- The dose is administered by "pilling" (for tablets).

- The dose is expelled or refused entirely (any dosage form).

The number of dose offerings may depend on the proposed dosing schedule:

- Single dose: one dose

- Repeated dose: full course of therapy

- Repeated monthly dose: at least 3 doses per animal

- Repeated daily dose: at least a week or the full course of therapy if less than a week

An example of a field study with 110 dogs was presented. The owners offered the dose twice daily for 7 days. The dogs voluntarily consumed $65 \%$ of the doses offered either by hand or in an empty bowl. The dogs voluntarily consumed $25 \%$ of the doses offered in food. The owners "pilled" $9 \%$ of the doses and were unable to administer $1 \%$ of the doses. These results could be summarized factually on the product labeling: "In a field study of 110 dogs that were each offered the label dose twice daily for 7 days, the dogs voluntarily consumed $65 \%$ of the doses offered as a treat and $25 \%$ of the doses offered in food; owners "pilled" $9 \%$ of the doses and were unable to administer $1 \% . "$

\section{REFERENCES}

1. Amidon, G. L.; Lennernäs, H.; Shah, V. P.; Crison, J. R. A Theoretical Basis for a Biopharmaceutic Drug Classification: The Correlation of in Vitro Drug Product Dissolution and in Vivo Bioavailability. Pharm. Res. 1995, 12 (3), 413-420. DOI: 10.1023/A:1016212804288.

2. Martinez, M.; Larry Augsburger, L.; Johnston, T.; Jones, W. W. Applying the Biopharmaceutics Classification 
System to veterinary pharmaceutical products: Part I: Biopharmaceutics and formulation considerations. Adv. Drug Delivery Rev. 2002, 54 (6), 805-824. DOI: 10.1016/ S0169-409X(02)00070-4.

3. Martinez, M.; Amidon, G.; Clarke, L.; Jones, W. W.; Mitra, A.; Riviere, J. Applying the biopharmaceutics classification system to veterinary pharmaceutical products. Part II. Physiological considerations. Adv. Drug Delivery Rev. 2002, 54 (6), 825-850. DOI: 10.1016/ S0169-409X(02)00071-6.

4. Martinez, M. N.; Papich, M. G.; Riviere, J. L. Veterinary Application of In Vitro Dissolution Data and the Biopharmaceutics Classification System. Pharm. Forum 2004, 30 (6).

5. Papich, M. G.; Martinez, M. N. Applying Biopharmaceutical Classification System (BCS) Criteria to Predict Oral Absorption of Drugs in Dogs: Challenges and Pitfalls. AAPSJ. 2015, 17 (4), 948-964. DOI: 10.1208/ s12248-015-9743-7.

6. Apley, M.; Crist, B.; Gonzalez, M. A.; Hunter, R. P.; Martinez, M. N.; Modric, S.; Papich, M. G.; Parr, A. F.; Riviere, J. E.; Marques, M. R. C. Solubility Criteria for Veterinary Drugs. Pharm. Forum 2012, 38 (4).

7. Apley, M.; Crist, B.; Gonzalez, M.; Hunter, R. P.; Martinez, M. N.; Modric, S.; Papich, M. G.; Parr, A. F.; Riviere, J. E.; Marques, M. R. C. Solubility Criteria for Veterinary Drugs-Workshop Report. Pharm. Forum 2013, 39 (4).

8. Apley, M.; Crist, G. B.; Fellner, V.; Gonzalez, M. A.; Hunter, R. P.; Martinez, M. N.; Messenheimer, J. R.; Modric, S.; Papich, M. G., Parr, A. F.; Riviere, J. E.; Marques, M. R. C. Determination of Thermodynamic Solubility of Active Pharmaceutical Ingredients for Veterinary Species: A New USP General Chapter. Pharm. Forum 2015, 41 (3).

9. Mudie, D. M.; Murray, K.; Hoad, C. L.; Pritchard, S. E.; Garnett, M. C.; Amidon, G. L.; Gowland, P. A.; Spiller, R. C.; Amidon, G. E.; Marciani, L. Quantification of Gastrointestinal Liquid Volumes and Distribution Following a $240 \mathrm{~mL}$ Dose of Water in the Fasted State. Mol. Pharmaceutics 2014, 11 (9), 3039-3047. DOI: 10.1021/mp500210c.

10. Martinez, M. N.; Papich, M. G. Factors Influencing the Gastric Residence of Dosage Forms in Dogs. J. Pharm. Sci. 2009, 98 (3), 844-860. DOI: 10.1002/jps.21499.

11. Interspecies Differences. National Institute of Public Health and the Environment (Netherlands) Web site http://www.interspeciesinfo.com (accessed Jan 15, 2017).

12. Johnson, M. C.; Devine, A. A.; Ellis, J. C.; Grunden, A. M.; Fellner, V. Effects of antibiotics and oil on microbial profiles and fermentation in mixed cultures of ruminal microorganisms. J. Dairy Sci. 2009, 92 (9), 4467-4480. DOI: 10.3168/jds.2008-1841.
13. <711> Dissolution. In The United States Pharmacopeia and National Formulary USP 39-NF 34; The United States Pharmacopeial Convention, Inc.: Rockville, MD, 2016.

14. $<724>$ Drug Release. In The United States Pharmacopeia and National Formulary USP 39-NF 34; The United States Pharmacopeial Convention, Inc.: Rockville, MD, 2016.

15. Dissolution Testing of Immediate Release Solid Oral Dosage Forms; Guidance for Industry; U.S. Department of Health and Human Services, Food and Drug Administration, Center for Drug Evaluation and Research (CDER), U.S. Government Printing Office: Washington, DC, 1997.

16. Dissolution Methods. U.S. Food and Drug Administration Web site http://www.accessdata.fda.gov/scripts/cder/ dissolution/ (accessed Jan 11, 2017).

17. USP Dissolution Methods Database. U.S. Pharmacopeial Convention Web site http://www.usp.org/usp-nf/ overview/compendial-tools/usp-dissolution-methodsdatabase (accessed Jan 11, 2017).

18. <1092> The Dissolution Procedure: Development and Validation. In The United States Pharmacopeia and National Formulary USP 39-NF 34; The United States Pharmacopeial Convention, Inc.: Rockville, MD, 2016.

19. <1152> Animal Drugs for Use in Animal Feeds. In The United States Pharmacopeia and National Formulary USP 39-NF 34; The United States Pharmacopeial Convention, Inc.: Rockville, MD, 2016.

20. Priddy, T. S.; Roush, R. R.; Bryson, L.; Folger, M. Characterization of the In Vitro Drug Exchange Profile of a Modified-Release Parenteral Solution for Veterinary Use. Dissolution Technol. 2017, 24 (1), 6-12. DOI: 10.14227/DT240117P6.

21. Orally Disintegrating Tablets; Guidance for Industry; U.S. Department of Health and Human Services, Food and Drug Administration, Center for Drug Evaluation and Research (CDER), U.S. Government Printing Office: Washington, DC, 2008.

22. Tablet Scoring: Nomenclature, Labeling, and Data for Evaluation; Guidance for Industry; U.S. Department of Health and Human Services, Food and Drug Administration, Center for Drug Evaluation and Research (CDER), U.S. Government Printing Office: Washington, DC, 2013.

23. Effectiveness of Anthelmintics: Specific Recommendations for Canine VICH GL19; Guidance for Industry; U.S. Department of Health and Human Services, Food and Drug Administration, Center for Veterinary Medicine, U.S. Government Printing Office: Washington, DC, 2002. 